\title{
Protocol for a Respondent-Driven Sampling Study Exploring the Roles of Peer Norms in HIV-Related Practices of Gay Men*
}

\author{
Iryna Zablotska ${ }^{1 \#,}$ John de Wit ${ }^{2}$, Graham Brown ${ }^{3,4}$, Bruce Maycock ${ }^{4}$, Christopher Fairley ${ }^{5}$, \\ Michelle McKechnie ${ }^{1}$, Garrett Prestage ${ }^{1,3}$
}

\begin{abstract}
${ }^{1}$ The Kirby Institute, University of New South Wales, Paddington, Australia; ${ }^{2}$ National Centre in HIV Social Research, University of New South Wales, Paddington, Australia; ${ }^{3}$ The Australian Research Centre in Sex, Health and Society, La Trobe University, Melbourne, Australia; ${ }^{4}$ Curtin University, Bentley, Australia; ${ }^{5}$ Melbourne School of Population Health, University of Melbourne and Melbourne Sexual Health Centre, Melbourne, Australia.

Email: "izablotska@kirby.unsw.edu.au
\end{abstract}

Received July 25 ${ }^{\text {th }}, 2013$; revised August 24 $4^{\text {th }}, 2013$; accepted August $29^{\text {th }}, 2013$

Copyright (c) 2013 Iryna Zablotska et al. This is an open access article distributed under the Creative Commons Attribution License, which permits unrestricted use, distribution, and reproduction in any medium, provided the original work is properly cited.

\begin{abstract}
Introduction: Sexual behaviour among gay and other men who have sex with men (GMSM) is influenced by the shared understanding of HIV/STI risk and peer norms regarding sexual behaviour. However, there is little research evidence to support this relationship. The unique geographic spread of Australian communities, the history of Australian HIV epidemic and the different patterns in risky sexual practices and HIV diagnoses across Australia present an opportunity to explore the role of social norms and GMSM behaviours in shaping different patterns of HIV epidemics. We describe the protocol of the study of contemporary norms in networks and communities of GMSM (CONNECT) which investigates gay community behavioural norms and practices. Methods: CONNECT is aimed to 1) identify the patterns of connections between individuals in GMSM communities and assess how they shape HIV-related behaviours; 2) describe the relationship between social norms and sexual practices; and 3) compare the norms and patterns of behaviour in geographically and epidemiologically distinct GMSM populations in three Australian states New South Wales, Victoria and Western Australia, in order to identify local community norms and barriers to effective HIV prevention. This quantitative cross-sectional study uses respondent-driven sampling (RDS) for recruitment. Results: Analyses will be carried out on individual and community level and will investigate the relationship between community-level behavioural norms and associated practices of individuals. Conclusion: CONNECT is anticipated to inform HIV prevention services to better target and increase the effectiveness of the current health promotion for GMSM in the context of increasing HIV/STI incidence.
\end{abstract}

Keywords: HIV; GMSM; Networks; Behaviour; Community Norms

\section{Introduction}

Sexual contact between gay and other men who have sex with men (GMSM) accounts for $85 \%$ of newly acquired HIV infections and is the most important HIV transmission route in Australia [1]. Therefore, GMSM are the highest priority group for HIV prevention [2].

\footnotetext{
*Funding: the Kirby Institute and the National Centre in HIV Social Research receive project funding from the Australian Government Department of Health and Ageing. The CONNECT study is funded by the Project Grant from the National Health and Medical Research Council, Australia (NHMRC 630547).

"Corresponding author.
}

Despite Australia's apparent success in reducing new HIV diagnoses in 1990s, there has been a steady increase in the annual number of new HIV cases nationally since 1999 [3]. Similar to worldwide trends [4-6], the resurgent HIV epidemic among GMSM has been accompanied by increasing rates of STI [7]. During the last decade, there have been marked changes in the patterns of the HIV epidemic across Australian jurisdictions, with a steady upward trends being recorded in all states, except for New South Wales (NSW) - the state with the highest number of newly diagnosed HIV infection [3,7].

Sexual behaviours among GMSM are known to be as- 
sociated with HIV infection, and unprotected anal intercourse (UAI) is the practice of the greatest risk [8]. Steady increases in UAI among GMSM, reported in recent years in Australia and worldwide, have been linked to the decreasing perceptions of risk [9], more frequent reliance on risk reduction practices, particularly serosorting, rather than on condom use $[8,10,11]$, improved survival and growing numbers of HIV-positive GMSM $[8,9,12]$, and changes in social and sexual networking. Recent increases in UAI among GMSM, including UAI with serodiscordant casual partners, have been linked to the increasing HIV/STI incidence $[3,8,13,14]$.

In Australia, trends in sexual behaviour across the states and territories mirror the HIV diagnoses trends and both differ across jurisdictions $[13,15]$. These differences in trends may be due to social differences among jurisdictions [16], the size and diversity of gay communities [12], the resources and the scale of the health promotion campaigns in the states [17], differences in background HIV prevalence [18], mixing of HIV positive and negative GMSM, and the patterns of risk management by both HIV positive and negative men. It has been assumed that GMSM communities across Australia differ with respect to their size [12] and culture. However, there has been limited research into what effect these differences may have on the interactions of individuals within communities and on their HIV/STI risk and risk reduction norms and practices $[19,20]$.

It is known that sexual behaviour among GMSM is influenced by the shared understanding of HIV/STI risk and shared norms regarding sexual behaviour in GMSM communities [21]. Risk reduction is more likely to occur if perceived community norms are supported and accepted. Currently, little is known about norms with respect to HIV/STI risk and risk reduction behaviours in GMSM communities, how GMSM understand community norms, and whether GMSM with different levels and types of connection to the gay community perceive behavioural norms in their own different ways. Previous studies found that the use of condoms was associated with men's attachment to the gay community, while socio-economic differences mediated this relationship [22]. However, it remains unclear how attachment to gay community affects behavioural norms and how norms are transmitted within and across different strata of GMSM and translated into sexual practices.

The unique geographic spread of Australian society and GMSM communities, the history of HIV epidemic and HIV prevention work across Australia, as well as different patterns in HIV diagnoses and trends in high risk sexual practices present an opportunity to explore and describe the role of social norms and individual behaviours in shaping different patterns in HIV epidemic across Australia. The study of contemporary norms in networks and communities of GMSM (CONNECT) was designed to elucidate these knowledge gaps and help to better understand why increasing efforts in HIV prevention during the last decade have been largely unable to reduce risky practices and the growing HIV incidence among GMSM.

\section{Methods}

\subsection{Theoretical Background}

The design and implementation of this study is guided by the current understanding of the central role of social processes in HIV prevention $[23,24]$. First, the study investigates behaviours and risk as the products of dynamic social interaction rather than "a static individual" phenomena. It is designed to take into account the context, negotiation and the different socially-constructed meanings that behaviour and risk may have in specific situations $[23,25,26]$, as well as the reciprocal influence between the context and the individual [27]. Understanding the interaction between individuals and their context is critical to the impact of the prevention interventions beyond the individual to the community level, and are vital to sustainable behaviour change [28]. Second, the CONNECT study investigates the social norms and beliefs among participants about how people should act in a given context, particularly the descriptive and subjective norms regarding sexual behaviours and HIV/STI in their peer networks $[29,30]$.

\subsection{Hypotheses}

The CONNECT study is driven by three key hypotheses:

1) HIV/STI sexual risk and risk reduction behaviours of individual GMSM depend on the behaviours of other GMSM with whom they interact, both sexually and socially; thus, the patterns of connections between individual GMSM are likely to have an effect on the overall levels of risk and risk reduction behaviours among GMSM.

2) GMSM with higher degrees of attachment to mainstream gay communities are more supportive of descriptive and subjective norms in their peers related to safe sex and are more likely to consistently maintain safe sex practices that conform to these norms.

3) The patterns of connections and behavioural norms of the geographically distinct populations of GMSM may influence the patterns of behaviour and the adoption of safe sex messages in local GMSM networks resulting in differing HIV notification trends in the Australian states.

\subsection{Recruitment Methods}

The choice of recruitment methodology to address these 
hypotheses is important. Traditional epidemiological approaches see individuals, with their characteristics and behaviours, as independent of each other [31] and ignore the effect of connections between individuals. Therefore, they miss to acknowledge larger social forces that drive different patterns of behaviour. If models of HIV/STIrelated behaviours were to incorporate patterns of social and sexual interactions between persons in a population, they would need to use methods that allow the tracking of connections between individuals, such as peer-referral methods.

Peer-referral methods have significantly developed recently, particularly respondent-driven sampling (RDS) [32]. Developed and tested by the US Centers for Disease Control, this methodology has been successfully used among GMSM worldwide and has been used in HIV/STI research to address the issues of hidden populations [33], the role of networks in infectious disease spread [34], sexual behaviours [35], and epidemic modelling [36]. The application details and effectiveness of RDS have been published [33].

Although RDS methodology was developed and largely used to capture representative samples of populations and recruit hard-to-recruit groups, this is not the only reason we used this recruitment methodology. We employed RDS mainly to explore and explain the role of connections between individuals in shaping personal net-works and community behavioural norms within these networks. Stringent confidentiality safeguards of this method also provide a bonus benefit for studies of sexual behaviours, like ours, which use peer-referral [37].

Since this is the first study among Australian GMSM to use RDS methodology for recruitment, the methodology was successfully pretested in a pilot study of 100 GMSM in Sydney, NSW.

The CONNECT study is aimed to 1) identify the patterns of connections between individuals in communities GMSM and assess how they shape HIV related behaviours; 2) describe the relationship between social norms and sexual practices among GMSM (particularly men with differing degrees of engagement with GMSM communities); and 3) compare the patterns of behaviour in geographically and epidemiologically distinct populations of GMSM in three Australian states New South Wales, Victoria and Western Australia, in order to identify local barriers to effective HIV prevention. In accomplishing these aims, the CONNECT study is anticipated to inform state and local HIV prevention services in Australia to better target and, thus, increase the effectiveness of the current health promotion for GMSM in the context of increasing HIV/STI incidence.

CONNECT is a cross-sectional quantitative study conducted in three geographically distinct, diverse and large gay communities in Sydney (NSW), Melbourne (Victoria) and Perth (WA). Eligible to participate in this study are men aged 18 years or older who live in proximity to any of the three research sites (state capital cities Sydney, Melbourne and Perth) or participate in the life of local GMSM community and acknowledge having had sex with another man during the preceding 12 months. Data collection employs a two stage approach. As stage 1, a mapping of the local GMSM communities was conducted to enlist local gay community groups, networks and organizations. The mapping of gay communities in Melbourne, VIC, was conducted as a separate study in 2009 [38] and other mapping exercises were conducted in Sydney, NSW and Perth, WA at the start of the CONNECT study in 2010, in preparation to the RDS recruitment. The mapping of gay communities in the three study sites has provided information to better understand the size, local context and structure of GMSM groups and networks. It also served as a preparation for further RDS recruitment of study participants in stage 2 . Based on the results of the mapping, primary RDS participants are selected to start recruitment in various community groups and venues.

\subsection{Data Collection}

Each of the primary participants completes the study survey and receives up to three referral coupons with unique ID numbers to refer only up to three peers to the study. They are asked to invite their friends and/or sex partners and receive a referral coupon with a unique identifying number for each of the referrals. The coupons also contain detail information on how prospective participants can contact the study recruitment officer in their respective research site and enrol into the study.

To increase awareness about the study, it is promoted in local GMSM community press, community organisations and in the popular local GMSM community venues identified during the mapping stage. Incentives, according to previous research, have been essential to maximise referrals for data collection efficiency [39]. However, this study does not use the individual incentive for each participant as our pilot study showed it would not be necessary and would not have an effect on recruitment. Instead, to encourage long wave recruitment, a substantial prize is offered to a winner of a lottery among the participants of the largest recruitment wave.

Recruitment and data collection is conducted by trained data collection officers (DCOs, one per each research site). Confidentiality is ensured by using 1 ) delinked data collection; 2) self-administered online questionnaire developed in Survey Gizmo, with password protected access to the dataset available only to the study project manager; 3) unique study identifying numbers 
based on the study referral process rather than personal identifying information; 4) encrypted data storage on secure computers; and 5) adequate training of all project personnel. We have further developed the recruitment method by using unique electronic referral coupons issued by DCOs, which participants could share with their referrals online.

Data are collected using a standard structured questionnaire about 30 - 40 minutes long which covers a broad range of HIV/STI related issues (see insert 1 for more detail).

To our knowledge this is the first RDS recruitment study which utilizes both online recruitment directed by project officers and online data collection.

\section{Insert 1. Content of the CONNECT online ques- tionnaire:}

Section I. Personal information: socio-demographics; sexual practices in the last 6 months with regular and casual sex partners, HIV disclosure; details of the recent sexual encounters, drug use; HVI/STI testing practices, attachment to gay community; size of personal network.

Section II. HIV status and STI incidence will be self-reported. We recognise the importance of serostatus verification, but the use of self-reported serostatus in this study is justifiable for two reasons: 1) this study is focused on GMSM behaviours, and these are determined by what individuals know or believe to be their serostatus; 2) our previous work shows a high prevalence and frequency of HIV testing and a high correlation between self-reported and actual serostatus among Australian GMSM [40]. Very few men in this study would indeed be HIV positive while not knowing it; thus, the high cost of HIV testing and counselling for the total sample would not be justified in this particular project.

Section III. Information about the referee: type of connection (social or sexual), sexual relationship if any (regular or casual partner), closeness of connection (how often they have been meeting in the past 6 months and in what circumstances), discussion of HIV and risk reduction strategies, perception of the contacts' expectations of how the respondent should act (subjective norms) and perceptions of HIV/STI risk and risk reduction behaviour of the contacts (descriptive norms).

Section IV. Community-level information: subjective and descriptive norms and beliefs about HIV/ STI risk and risk reduction practices; perceptions of the knowledge about and attitudes towards HIV prevention methods, and barriers to safe sex practices in their respective personal networks and communities.

\subsection{Data Analyses}

Quantitative data collected by this study will be analysed using RDS analytic methods [41] as well as conventional statistical methods. Analyses will be carried out in several directions. First, a significant body of behavioural research and surveillance has already been developed in Australia, and time-location sampling has been widely used for recruitment of gay men. This is the first study to use RDS methodology in Australian gay communities. Therefore, the sample obtained by RDS recruitment will be thoroughly compared to samples from behavioural surveillance surveys and other studies to assess its representativeness and additive value for research among gay men.

Second, the main focus of this study is to explore the understanding of behavioural norms in gay communities and the effect of norms on sexual practices of individuals. As behaviours of individuals are not independent, analyses will explore the connections between individuals, their environment (that is their personal networks) and the shared understanding of norms and behaviours within these networks. Furthermore, they will focus on the shared understanding of norms and how these are translated into self-reported sexual and other practices associated with HIV/STI transmission. Analyses will also be conducted on the extent of concordance in perceived behavioural norms within dyads of participants connected by recruitment (some of these being sex partners). As a measure of diffusion of norms through the referral chains, the concordance in perceived behavioural norms will also be explored according to the degree of distance between participants within the chain of referral (direct connection, separated by one, two or more persons in the referral chains).

Third, one of the focuses of this study is on the comparison of individual-level behaviours and communitylevel behavioural norms across geographically distinct communities of gay men. Comparisons will be drawn across three Australian states involved in this research to elucidate the effect of local community norms on behaviour. This will contribute to the understanding of how local behavioural norms with respect to HIV risk and risk reduction are shaped.

\subsection{Ethics}

The study procedures have been approved by the Human Research Ethics Committees of the two participating Universities (University of New South Wales, Sydney and Curtin University, Perth, SA), as well as by key gay community organizations in each of the three research sites (AIDS Councils of NSW, Victoria and Western Australia). Significant effort was placed at the stage of the study design to ensure that the process of peer-refer- 
ral is combined with participant anonymity and confidentiality of collected data.

\section{Results}

Report on the study findings will be co-produced by the study advisory committee, whose membership represents the partnership of behavioural and social researchers, representatives of community organizations and policy makers from the Australian HIV sector. We previously reported the scarce research work as to the behavioural norms in gay communities. Better understanding of community behavioural norms may inform the development of more effective HIV/STI prevention and education interventions. Therefore, the findings of this study, much anticipated by HIV prevention programs, will be disseminated not only to academic audiences, but also widely presented both locally and nationally, in community and public health forums.

\section{Conclusion}

It is anticipated that the evidence collected by the CONNECT study will assist in improved targeting of HIV prevention and health promotion in Australia and elsewhere. We hope that this study will also contribute to the debate about the role of personal environment and broader community in shaping individual decision-making and practices which have bared on individual and community health.

\section{Acknowledgements}

The authors would like to acknowledge the key community partners the AIDS Council of Western Australian, Victorian AIDS Council and AIDS Council of New South Wales, as well and PLWHA organizations in each state, for their support to this study and close involvement in the implementation of this study.

\section{Contributors}

Iryna Zablotska, John de Wit, Graham Brown, Bruce Maycock, Christopher Fairley, Michelle McKechnie and Garrett Prestage assumed principal responsibility for the design and implementation of this study. All authors contributed to the preparation of and approved this protocol manuscript.

\section{Conflicts of Interest}

None were declared.

\section{REFERENCES}

[1] HIV, "Viral Hepatitis and Sexually Transmissible Infec- tions in Australia,” Annual Surveillance Report 2010, The University of New South Wales, Sydney, 2010.

[2] Australian Government Department of Health and Aging, "6th National HIV Strategy (2010-2013)," Australian Government Department of Health and Aging, Canberra, 2010.

[3] R. J. Guy, A. McDonald, M. Bartlett, J. Murray, C. M. Giele, T. M. Davey, et al., "HIV Diagnoses in Australia: Diverging Epidemics within a Low-Prevalence Country," Medical Journal of Australia, Vol. 187, No. 8, 2007, pp. 437-440.

[4] N. MacDonald, S. Dougan, C. A. McGarrigle, K. Baster, B. D. Rice, B. G. Evans, et al., "Recent Trends in Diagnoses of HIV and Other Sexually Transmitted Infections in England and Wales among Men Who Have Sex with Men,” Sexually Transmitted Infections, Vol. 80, No. 6, 2004, pp. 492-497. http://dx.doi.org/10.1136/sti.2004.011197

[5] U. Marcus, L. Voss, C. Kollan and O. Hamouda, "HIV Incidence Increasing in MSM in Germany: Factors Influencing Infection Dynamics,” Eurosurveillance, Vol. 11, No. 9, 2006, pp. 157-160.

[6] R. J. Wolitski, R. O. Valdiserri, P. H. Denning and W. C. Levine, "Are We Headed for a Resurgence of the HIV Epidemic among Men Who Have Sex with Men?” American Journal of Public Health, Vol. 91, No. 6, 2001, pp. 883-888. http://dx.doi.org/10.2105/AJPH.91.6.883

[7] A. McDonald, "HIV/AIDS, Viral Hepatitis and Sexually Transmissible Infections in Australia: Annual Surveillance Report 2008," Australian Institute of Health and Welfare, Canberra, 2008.

[8] J. Elford, "Changing Patterns of Sexual Behaviour in the Era of Highly Active Antiretroviral Therapy," Current Opinion in Infectious Diseases, Vol. 19, No. 1, 2006, pp. 26-32. http://dx.doi.org/10.1097/01.qco.0000199018.50451.e1

[9] N. Crepaz, T. A. Hart and G. Marks, "Highly Active Antiretroviral Therapy and Sexual Risk Behavior: A MetaAnalytic Review,” JAMA, Vol. 292, No. 2, 2004, pp. 224236. http://dx.doi.org/10.1001/jama.292.2.224

[10] L. Mao, J. M. Crawford, H. J. Hospers, G. P. Prestage, A. E. Grulich, J. M. Kaldor, et al., “'Serosorting' in Casual Anal Sex of HIV-Negative Gay Men Is Noteworthy and Is Increasing in Sydney, Australia,” AIDS, Vol. 20, No. 8, 2006, pp. 1204-1206. http://dx.doi.org/10.1097/01.aids.0000226964.17966.75

[11] F. Jin, J. Crawford, G. P. Prestage, I. Zablotska, J. Imrie, S. C. Kippax, et al., "Unprotected Anal Intercourse, Risk Reduction Behaviours, and Subsequent HIV Infection in a Cohort of Homosexual Men,” AIDS, Vol. 23, No. 9, 2009, pp. 243-252.

http://dx.doi.org/10.1097/QAD.0b013e32831fb51a

[12] G. Prestage, J. Ferris, J. Grierson, R. Thorpe, I. Zablotska, J. Imrie, et al., "Homosexual Men in Australia: Population, Distribution and HIV Prevalence,” Sex Health, Vol. 5, No. 2, 2008, pp. 97-102. http://dx.doi.org/10.1071/SH07080

[13] I. B. Zablotska, G. Prestage, A. E. Grulich and J. Imrie, 
"Differing Trends in Sexual Risk Behaviours in Three Australian States: New South Wales, Victoria and Queensland, 1998-2006," Sex Health, Vol. 5, No. 2, 2008, pp. 125-130. http://dx.doi.org/10.1071/SH07076

[14] I. B. Zablotska, J. Crawford, J. Imrie, G. Prestage, F. Jin, A. Grulich, et al., "Increases in Unprotected Anal Intercourse with Serodiscordant Casual Partners among HIVNegative Gay Men in Sydney,” AIDS and Behavior, Vol. 13, No. 4, 2009, pp. 638-644. http://dx.doi.org/10.1007/s10461-008-9506-x

[15] I. B. Zablotska, G. Prestage, M. Middleton, D. Wilson and A. E. Grulich, "Contemporary HIV Diagnoses Trends in Australia Can Be Predicted by Trends in Unprotected Anal Intercourse among Gay Men,” AIDS, Vol. 24, No. 12, 2010, pp. 1955-1958. http://dx.doi.org/10.1097/QAD.0b013e32833bf74a

[16] "A Think Tank: Why Are HIV Notifications Flat in NSW 1998-2006?” Consensus Statement: NSW Health, 2007. http://www.health.nsw.gov.au/sexualhealth/Documents/H IV-consensus-statement.pdf

[17] S. Kippax, R. W. Connell, G. Dowsett and J. Crawford, "Sustaining Safe Sex: Gay Communities Respond to AIDS," The Falmer Press, London, Washington DC, 2008.

[18] G. Prestage, F. Jin, I. Zablotska, J. Imrie, J. M. Kaldor and A. E. Grulich, "Trends in HIV Prevalence among Homosexual and Bisexual Men in Eastern Australian States," Sex Health, Vol. 5, No. 2, 2008, pp. 103-107. http://dx.doi.org/10.1071/SH07074

[19] G. Brown, B. Maycock, G. Prestage and P. V. Ven, "Sex in Two Cities-Gay Men, Risk and HIV in Perth and Sydney," Asia-Pacific Journal of Public Health/Asia-Pacific Academic Consortium for Public Health, Vol. 16, No. 2, 2004, pp. 3-8.

[20] M. S. Rowe and G. W. Dowsettz, "Sex, Love, Friendship, Belonging and Place: Is There a Role for 'Gay Community’ in HIV Prevention Today?” Culture, Health \& Sexuality, Vol. 10, No. 4, 2008, pp. 329-344. http://dx.doi.org/10.1080/13691050701843098

[21] S. Kippax and K. Race, "Sustaining Safe Practice: Twenty Years on,” Social Science \& Medicine, Vol. 57, No. 1, 2003, pp. 1-12. http://dx.doi.org/10.1016/S0277-9536(02)00303-9

[22] R. W. Connell, G. W. Dowsett, P. Rodden, M. D. Davis, L. Watson and D. Baxter, "Social Class, Gay Men and AIDS Prevention," Australian Journal of Public Health, Vol. 15, No. 3, 1991, pp. 178-189. http://dx.doi.org/10.1111/j.1753-6405.1991.tb00332.x

[23] G. Hart and M. Boulton, "Sexual Behaviour in Gay Men: towards a Sociology of Risk,” In: P. Aggleton, P. Davis and G. Hart, Eds., AIDS: Safety, Sexuality and Risk, Taylor and Francis Ltd., London, 1995.

[24] T. Rhodes, "Risk Theory in Epidemic Times: Sex, Drugs and the Social Organisation of Risk Behaviour," Sociology of Health \& Illness, Vol. 19, No. 2, 1997, p. 227. http://dx.doi.org/10.1111/1467-9566.00047

[25] S. Kippax, S. Slavin, J. Ellard, O. Hendry, J. Richters, A. Grulich, et al., "Seroconversion in Context," AIDS Care, Vol. 15, No. 6, 2003, pp. 839-852.

\section{http://dx.doi.org/10.1080/09540120310001618685}

[26] R. H. Jones and C. N. Candlin, "Constructing Risk across Timescales and Trajectories: Gay Men's Stories of Sexual Encounters," Health, Risk and Society, Vol. 5, No. 2, 2003, pp. 199-213.

http://dx.doi.org/10.1080/1369857031000123966

[27] A. Bandura, "Social Foundations of Thought and Action: A Social Cognitive Theory,” Prentice-Hall, Englewood Cliffs, 1986.

[28] E. Rogers, "Diffusion of Innovations," Free Press, New York, 1983.

[29] H. Liu, H. Liu, Y. Cai, A. G. Rhodes and F. Hong, "Money Boys, HIV Risks, and the Associations between Norms and Safer Sex: A Respondent-Driven Sampling Study in Shenzhen, China,” AIDS and Behavior, Vol. 13, No. 4, 2009, pp. 652-662.

[30] J. L. Peterson, R. Rothenberg, J. M. Kraft, C. Beeker and R. Trotter, "Perceived Condom Norms and HIV Risks among Social and Sexual Networks of Young African American Men Who Have Sex with Men,” Health Education Research, Vol. 24, No. 1, 2009, pp. 119-127. http://dx.doi.org/10.1093/her/cyn003

[31] J. S. Koopman and J. W. Lynch, "Individual Causal Models and Population System Models in Epidemiology," American Journal of Public Health, Vol. 89, No. 8, 1999, pp. 1170-1174. http://dx.doi.org/10.2105/AJPH.89.8.1170

[32] D. Heckathorn, S. Semaan, R. Broadhead and J. Hughes, "Extensions of Respondent-Driven Sampling: A New Approach to the Study of Injecting Drug Users Aged 18 25,” AIDS and Behavior, Vol. 6, No. 1, 2002, pp. 55-67. http://dx.doi.org/10.1023/A:1014528612685

[33] R. Magnani, K. Sabin, T. Saidel and D. Heckathorn, "Review of Sampling Hard-To-Reach and Hidden Populations for HIV Surveillance,” AIDS, Vol. 19, Suppl. 2, 2005, pp. S67-S72.

http://dx.doi.org/10.1097/01.aids.0000172879.20628.e1

[34] A. S. Klovdahl, "Social Networks and the Spread of Infectious Diseases: The AIDS Example,” Social Science \& Medicine, Vol. 21, No. 11, 1985, pp. 1203-1216. http://dx.doi.org/10.1016/0277-9536(85)90269-2

[35] C. A. Latkin, V. Forman, A. Knowlton and S. Sherman, "Norms, Social Networks, and HIV-Related Risk Behaviors among Urban Disadvantaged Drug Users,” Social Science \& Medicine, Vol. 56, No. 3, 2003, pp. 465-476. http://dx.doi.org/10.1016/S0277-9536(02)00047-3

[36] S. M. Goodreau, “Advances in Exponential Random Graph (p*) Models Applied to a Large Social Network,” Social Networks, Vol. 29, No. 2, 2007, pp. 231-248. http://dx.doi.org/10.1016/j.socnet.2006.08.001

[37] A. Klovdahl, "Social Network Research and Human Subjects Protection: Towards More Effective Infectious Disease Control," Social Networks, Vol. 27, No. 2, 2005, pp. 119-137. http://dx.doi.org/10.1016/j.socnet.2005.01.006

[38] A. Smith, J. Grierson, H. von Doussa, M. Pitts and T. Clement, “Mapping Gay Men's Communities,” Monograph Number 73, Australian Research Centre in Sex, Health and Society, La Trobe University, Melbourne, 2009. 
[39] M. Xiridou, R. Geskus, J. de Wit, R. Coutinho and M. Kretzschmar, "The Contribution of Steady and Casual Partnerships to the Incidence of HIV Infection among Homosexual Men in Amsterdam,” AIDS, Vol. 17, No. 7, 2003, pp. 1029-1038. http://dx.doi.org/10.1097/00002030-200305020-00012

[40] I. G. Stolte, J. B. de Wit, A. van Eeden, R. A. Coutinho and N. H. Dukers, "Perceived Viral Load, but Not Actual HIV-1-RNA Load, Is Associated with Sexual Risk Be- haviour among HIV-Infected Homosexual Men,” AIDS, Vol. 18, No. 14, 2004, pp. 1943-1949. http://dx.doi.org/10.1097/00002030-200409240-00010

[41] M. J. Salganik and D. Heckathorn, "Sampling and Estimation in Hidden Populations Using Respondent Driven Sampling,” Sociological Methodology, Vol. 34, No. 1, 2004, pp. 193-239. http://dx.doi.org/10.1111/j.0081-1750.2004.00152.x 